\title{
Down-regulation of osteopontin inhibits metastasis of hepatocellular carcinoma cells via a mechanism involving MMP-2 and uPA
}

\author{
RONG-XIN CHEN ${ }^{1,2}$, YUN-HONG XIA ${ }^{1,2}$, TONG-CHUN XUE ${ }^{1,2}$, \\ HONG ZHANG ${ }^{3}$ and SHENG-LONG $\mathrm{YE}^{1,2}$ \\ ${ }^{1}$ Liver Cancer Institute and Zhongshan Hospital, Fudan University; ${ }^{2}$ Key Laboratory for Carcinogenesis \\ and Cancer Invasion, the Chinese Ministry of Education, Shanghai 200032, P.R. China; \\ ${ }^{3}$ Isis Pharmaceuticals, Carlsbad, CA 92008, USA
}

Received September 1, 2010; Accepted October 4, 2010

DOI: $10.3892 /$ or.2010.1116

\begin{abstract}
Osteopontin (OPN) has an important role in hepatocellular carcinoma (HCC) progression and metastasis. This study was to investigate the therapeutic potential of inhibition of OPN expression. A 2'-O-methoxyethylribosemodified phosphorothioate antisense oligonucleotides (ASO) was used to knock-down OPN expression in the human metastatic HCC cell line HCCLM6 and in nude mice orthotopically implanted with HCCLM6 showing highly spontaneous lung metastasis. Furthermore, we assessed the metastatic potential of HCCLM6 cells in vitro and in vivo after ASO treatment. Treatment of HCCLM6 cells with OPN ASO inhibited OPN mRNA expression in a dose- and timedependent manner, whereas the control oligonucleotides had no effect. OPN ASO significantly suppressed migration and invasion of HCCLM6 cells in vitro. Specific suppression of OPN also inhibited matrix metalloproteinase 2 (MMP-2) and urokinase-type plasminogen activator (uPA) expression in HCCLM6 cells. In mice bearing orthotopical xenografts with HCCLM6, OPN inhibition following therapeutic treatment with OPN ASO significantly decreased lung metastases although tumor weight did not appear to be reduced. These
\end{abstract}

Correspondence to: Dr Sheng-Long Ye, Department of Hepatic Oncology, Liver Cancer Institute and Zhongshan Hospital, Fudan University, No. 180 Fenglin Road, Shanghai 200032, P.R. China E-mail: ye.shenglong@zs-hospital.sh.cn

Abbreviations: OPN, osteopontin; HCC, hepatocellular carcinoma; ASO, antisense oligonucleotides; MMP-2, matrix metalloproteinase 2; uPA, urokinase-type plasminogen activator; 2'-MOE, 2'-O-methoxyethyl; PI3K, phosphotidylinositol 3-kinase; NF-кB, nuclear factor $\kappa \mathrm{B}$; MMPs, matrix metalloproteinases; MAPK, mitogen-activated protein kinases; AP-1, activator protein-1

Key words: hepatocellular carcinoma, osteopontin, metastasis, antisense oligonucleotides findings suggest that OPN-targeted therapy may be a promising strategy for the treatment of HCC metastases.

\section{Introduction}

Hepatocellular carcinoma (HCC) is one of the most common and aggressive malignancies, ranking the third in the world and the second in China in cancer-related deaths (1). Despite improvements in the available treatments such as surgical resection, liver transplantation and locoregional therapy, HCC prognosis remains poor due to the high rate of recurrence or metastasis after therapy (2). Thus, it is critical to identify the molecule controlling the invasive and metastatic potential of HCC, which would provide new targets for intervention.

Results from gene profiling analysis indicate that osteopontin (OPN) is identified as the lead gene overexpressed in metastatic HCC (3). OPN has recently been shown to be overexpressed in a variety of cancers including colon, breast, lung, prostate, and pancreatic cancer (4). OPN contributes to tumor progression, especially tumor metastasis through the promotion of cell proliferation, migration and extracellular matrix invasion in vitro $(5,6)$. Specific inhibition of OPN signaling has also been shown to inhibit tumor progression and metastasis in other types of cancers (7). These findings suggest that OPN may be a novel drug target for therapeutic intervention in $\mathrm{HCC}$ progression and metastasis.

In our institute, human HCC cell line HCCLM6 with highly metastatic potential and the orthotopic model with spontaneous lung metastasis have been established previously (8), which is more reflective of HCC development and metastasis. Herein, we showed that inhibition of OPN using a specific 2'-O-methoxyethyl (2'-MOE)-modified antisense oligonucleotide (ASO) reduced cell migration and invasion of HCCLM6 cells in vitro. In addition, expression of matrix metalloproteinase 2 (MMP-2) and urokinase-type plasminogen activator (uPA) was reduced following OPN suppression. Moreover, the lung metastasis of HCCLM6 in nude mice treated with OPN ASO was effectively inhibited. Thus, the present results show that targeting OPN may have therapeutic potential in inhibiting HCC metastasis. 


\section{Materials and methods}

Cell culture and oligonucleotide treatment. Human HCC cell line HCCLM6 established at the Liver Cancer Institute, Zhongshan Hospital, Fudan University, Shanghai, China, was cultured in DMEM (Invitrogen, Carlsbad, CA) containing $10 \%$ fetal bovine serum (FBS) in $5 \% \mathrm{CO}_{2}$ at $37^{\circ} \mathrm{C}$. Oligonucleotides were 20 -mer in length with a phosphorothioate back-bone and five 2'-MOE-modified nucleotides at each end (underlined residues below). The 10 central bases were left unmodified to support RNase H-mediated cleavage of targeted mRNA. OPN ASO was designed to target the coding region of human OPN mRNA (GenBank NM_000582; 5'- $\underline{\text { ACTTC }}$ GGTTGCTGGCAGGTC-3'). The control oligonucleotides had the same chemical design except for the randomized sequence. Oligonucleotides were synthesized as previously described. For cell treatment, oligonucleotides premixed with Lipofectamine 2000 (Invitrogen) at $1.25 \mu \mathrm{g} / \mathrm{ml} / 100 \mathrm{nM}$ ASO in serum-free DMEM were added to cells $\left(37^{\circ} \mathrm{C}, 6 \mathrm{~h}\right)$. The oligonucleotide-lipid treatment was then replaced with culture medium and incubated for the indicated times.

RNA isolation and reverse transcription-PCR. Total RNA was extracted from cells using TRIzol reagent (Invitrogen) and reverse transcribed into single-stranded cDNA. PCR was done on cDNA using oligo(dT) priming and amplified with the primer pairs for a 436-bp fragment of OPN (forward primer 5'-GGACTCCATTGACTCGAACG-3' and reverse primer 5'-TAATCTGGACTGCTTGTGGC-3') and a 366-bp fragment of Glyceraldehyde-3-phosphate dehydrogenase (GAPDH) (forward primer 5'-ATCCCATCACCATCTT CCAG-3' and reverse primer 5'-GAGTCCTTCCACGATA CCAA-3'). Ten microliters of PCR product were analyzed on $2 \%$ agarose gels.

Western blotting. Cell lysate (50 $\mu \mathrm{g})$ was separated by standard SDS-PAGE and then transferred to PVDF membranes. The membranes were washed, blocked, and incubated with the specific primary antihuman antibodies against OPN (1:500; Santa Cruz), or glyceraldehyde-3-phosphate dehydrogenase (1:5000; Santa Cruz), followed by incubation with horseradish peroxidase-conjugated secondary antibodies. The reactions were detected by enhanced chemiluminescence assay.

Immunocytochemistry. Cells cultured on cover slides in sixwell plates $\left(1.0 \times 10^{5} /\right.$ well $)$ were fixed in acetone and then stained with monoclonal mouse anti-human OPN (1:100; Santa Cruz) after a 3\% hydrogen peroxide-methanol blocking solution. Following incubation with a horseradish peroxidaseconjugated secondary antibody (Dako), staining was developed using AEC chromogen substrate followed by counterstaining with hematoxylin.

Cell proliferation assay. The cellular proliferation of cells was measured by the MTT assay (Chemicon International, Inc.). Cells $\left(1 \times 10^{4}\right)$ were plated in 96 -well plates prior to treatment at the indicated concentrations. After incubation for 1-4 days, the cells were assayed at the indicated times following the manufacturer's protocol.
Flow cytometry. Cells were grown to subconfluence and treated with OPN ASO at the indicated concentration. The cells were trypsinized and fixed in $70 \%$ ethanol overnight and stained for $30 \mathrm{~min}$ with propidium iodide $(0.1 \mu \mathrm{g} / \mathrm{ml})$ followed by analysis.

Matrigel invasion assay and migration assay. The invasive ability of the transfected cells was determined by the Matrigel (BD Pharmingen) coated 24-well transwell chambers with upper and lower culture compartments separated by polycarbonate membranes with $8-\mu \mathrm{m}$ pore (Costar, NY, USA). The bottom chamber was filled with DMEM containing $10 \%$ FBS as a chemoattractant. The transfected cells $\left(1 \times 10^{5}\right)$ after treatment were seeded on the top chamber and incubated at $37^{\circ} \mathrm{C}$ with $5 \% \mathrm{CO}_{2}$. After $40 \mathrm{~h}$, the cells removed from the upper surface of the Matrigel by scrubbing with a cotton swab and cells that migrated to the underside of the membrane were stained with Giemsa (Sigma). Five high-power fields were counted and the mean number of cells per field was calculated. The migration assay was similar to the invasion assay, only without Matrigel. The experiments were carried out in triplicate.

Enzyme-linked immunosorbent assay (ELISA). Serum-free supernatants from $4 \times 10^{5}$ cells after treatment were measured for MMP-2, and UPA secretion by ELISA according to manufacturer's directions (MMP-2, MMP-9 ELISA kit from R\&D Systems, Minneapolis, MN; uPA ELISA kit from American Diagnostica Inc.). Absorbance was measured at $450 \mathrm{~nm}$ and $570 \mathrm{~nm}$ using a microplate spectrophotometer. Each assay was conducted in triplicate.

Gelatin zymography. Gelatin zymography was performed as previously described. Briefly, 3x105 HCCLM6 cells were plated in six-well tissue culture plates and treated with oligonucleotides at the indicated time. Then, media were replaced with serum-free DMEM for $24 \mathrm{~h}$ and the supernatant was collected. The samples were resolved in $10 \%$ gelatin (Invitrogen) and the gel was developed according to the manufacturer's instructions. The enzyme activity was visualized by staining the gel with Coomassie brilliant blue R250.

Therapeutic treatment of orthotopic HCCLM6 tumors in nude mice with OPN ASO. Male 6- to 8-week-old BALB/c nu/nu mice were obtained from the Shanghai Institute of Materia Medica, Chinese Academy of Sciences, Shanghai, China. Animals were maintained in accordance with the recommendations of the NIH Guidelines for Care and Use of Laboratory Animals. HCCLM6 ells were intrahepatically implanted into male BALB/c nude mice under sterile conditions as previously described. Mice were then randomly assigned into two groups ( $n=6 /$ each group) and treated by intraperitoneal injectecion (i.p.) with $50 \mathrm{mg} / \mathrm{kg}$ oligonucleotide in saline solution. On day 35 after implantation (i.e., 3 days after the last dose), mice were sacrificed to examine tumor growth and metastases. Blood, liver tumor, and lung tissues were collected. Formalin-fixed liver tumors were examined for OPN protein levels using immunohistochemistry methods. Lung tissues were evaluated for metastases 


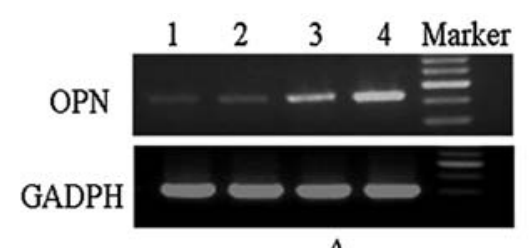

A

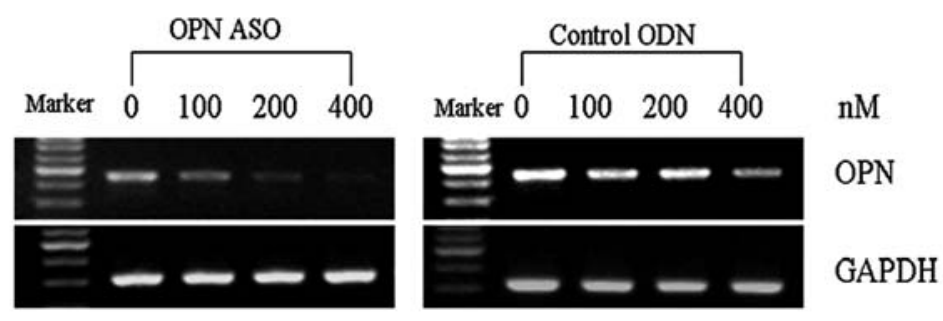

B

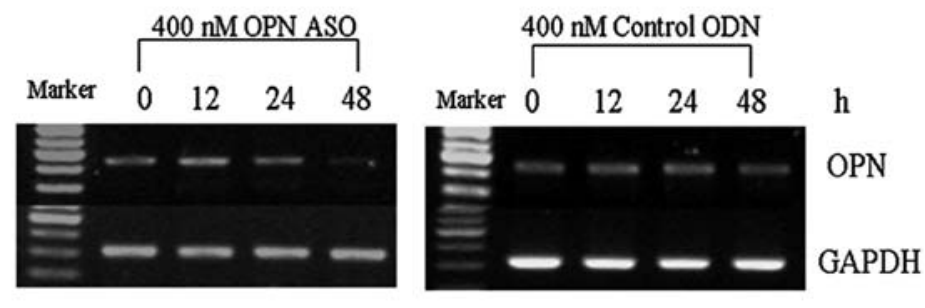

C

Figure 1. OPN mRNA expression was overexpressed in HCCLM6 cells and was inhibited by OPN ASO. (A) OPN mRNA expression of different cell lines was detected by RT-PCR. Lane 1, normal liver cell line L02; 2, non-metastatic HCC cell line SMMC-7721; 3, low metastatic HCC cell line MHCC97-L; 4, high metastatic HCC cell line HCCLM6. (B) OPN ASO inhibited OPN mRNA expression in a dose-dependent manner whereas control oligonucleotide (Control ODN) had no effect. (C) OPN ASO inhibited OPN mRNA expression at indicated concentrations (400 nM) in a time-dependent manner.

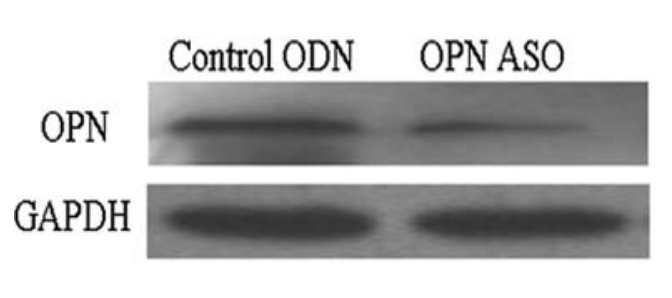

A

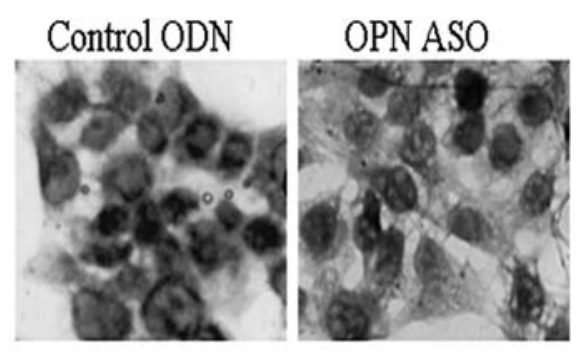

B

Figure 2. OPN protein expression was inhibited by OPN ASO in HCCLM6 cells. (A) OPN expression of HCCLM6 cells treated with OPN ASO (400 nM, 48 h) detected by Western blots. (B) Immunocytochemical staining of OPN in HCCLM6 cells treated with OPN ASO (400 nM, 48 h).

by sequential sections of paraffin-embedded tissue with $\mathrm{H} \& \mathrm{E}$ staining.

Statistical analysis. Statistical analyses were performed by the Statistical Package for the Social Sciences version 11.5 (SPSS, Inc., Chicago, IL). Data are expressed as the mean \pm SD, and analyzed using the two-tailed Student's t-test or the analysis of variance (ANOVA). The survival time of the animals was compared by the log-rank and Kaplan-Meier methods. The level of significance was set at $\mathrm{P}<0.05$.

\section{Results}

Knockdown of OPN expression in HCCLM6 cells by OPN ASO. HCCLM6 cells overexpressed OPN protein compared with low or non-metastatic HCC cell lines (Fig. 1A). Treatment of HCCLM6 cells with OPN ASO inhibited OPN mRNA expression detectable by RT-PCR in a dose- and timedependent manner, whereas the same 2'-MOE-modified, sequence-randomized oligonucleotides had no effect (Fig. 1B and $\mathrm{C}$ ), suggesting the presence of a sequence specific antisense activity. In agreement with RT-PCR results, OPN protein in HCCLM6 cells treated with OPN ASO was also reduced compared to control oligonucleotides (Fig. 2A). In addition, immunocytochemical staining revealed that OPN expression significantly decreased following OPN ASO treatment, but not by control oligonucleotides (Fig. 2B).

Effects of OPN down-regulation by OPN ASO on cell proliferation, apoptosis, migration and invasion of HCCLMG 

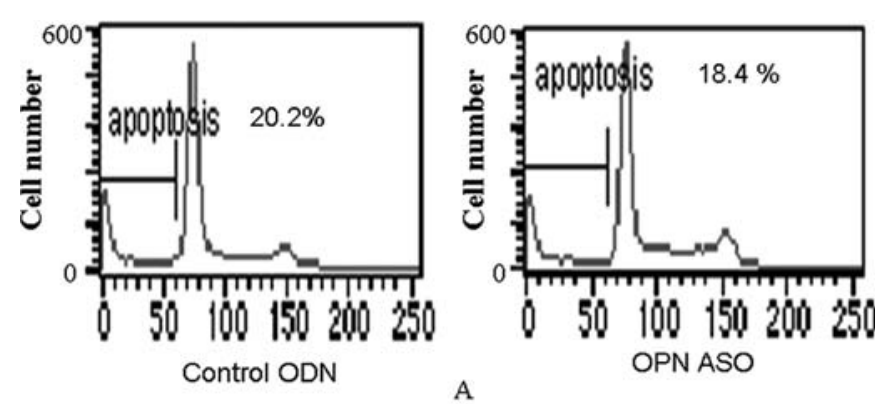

A

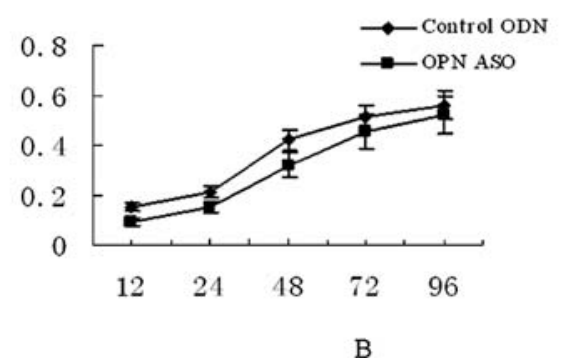

Figure 3. Effects of OPN suppression on the proliferation and apoptosis of HCCLM6 cells. HCCLM6 cells were treated with OPN ASO (400 nM, 48 h) or control oligonucleotide (Control ODN). (A) Flow cytometric analysis of apoptotic cells in HCCLM6 following propidium iodide staining. (B) The rate of proliferation in HCCLM6 cells treated with OPN ASO was not significantly different from that in Control ODN-treated cells.

cells in vitro. Effects of OPN knockdown by OPN ASO on the proliferation and apoptosis of HCCLM6 cells were measured by MTT assay and flow cytometry. Cell proliferation was not significantly inhibited (Fig. 3A) and apoptosis was not increased in the HCCLM6 cells administered with OPN ASO compared to those treated with control oligonucleotides (apoptotic rate: $18.4 \%$ vs. $20.2 \%$, Fig. 3B). As migratory and invasive behaviors are the indicators of the metastatic potential, we examined HCCLM6 migration and invasion in vitro using the transwell assay after OPN was down-regulated by OPN ASO. The numbers of HCCLM6 cells treated with OPN ASO migrating toward the conditioned medium or invading

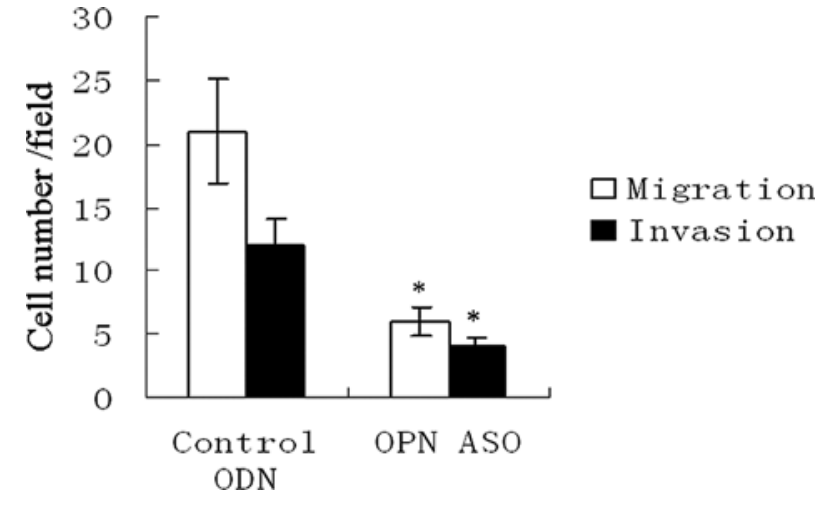

Figure 4. Inhibition of HCCLM6 migration and invasion in vitro by targeting OPN. HCCLM6 cells were treated with OPN ASO (400 nM, 48 h) or control oligonucleotide (Control ODN). Cell migratory and invasive ability was measured by transwell assay. Statistically significant difference of migration and invasion in OPN ASO-treated HCCLM6 cells and Control ODN-treated cells, ${ }^{*} \mathrm{P}<0.05$.

through the Matrigel were significantly fewer than those treated with control oligonucleotide (Migration assay: $6.80 \pm 2.49$ vs. $20.60 \pm 3.65, \mathrm{P}<0.05$; Invasion assay: $4.40 \pm 1.67$ vs. $11.60 \pm 3.36$, Fig. 4 ), suggesting that the migration and invasion potential of HCCLM6 cells was decreased after inhibition of OPN.

The decreased expression of MMP-2 and $\mathrm{PPA}$ following $O P N$ ASO treatment. Since OPN regulated HCCLM6 cells migration and invasion in vitro, we examined the expression of MMP-2 and UPA involved in the digestion of extracellular matrices, penetration of stroma, and establishment of metastasis. Following OPN ASO treatment, MMP-2 and uPA levels in culture supernatants of HCCLM6 cells were significantly reduced (MMP-2 level: $18.07 \pm 3.28 \mathrm{ng} / \mathrm{ml}$ vs. $40.08 \pm 7.74 \mathrm{ng} / \mathrm{ml}, \mathrm{P}<0.05$; uPA level: $1.69 \pm 0.32 \mathrm{ng} / \mathrm{ml} \mathrm{vs}$. $3.85 \pm 0.54 \mathrm{ng} / \mathrm{ml}, \mathrm{P}<0.05$, Fig. 5A). To furthermore determine if the reduced invasion of HCCLM6 treated by OPN ASO

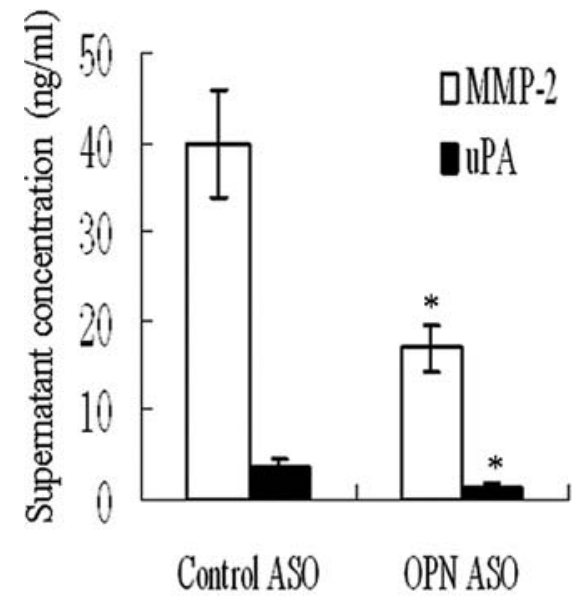

A

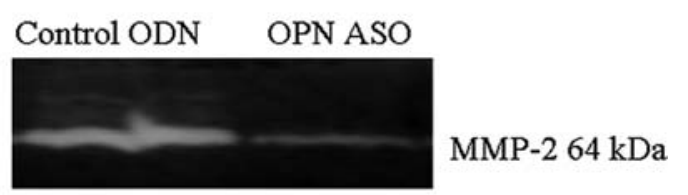

B

Figure 5. MMP-2 and uPA involved in the inhibitory effects of ASO OPN on the invasion and metastasis of HCCLM6 cells. (A) The levels of MMP-2 and uPA measured by ELISA analysis were significantly decreased in the medium of OPN ASO-treated HCCLM6 cells. (B) In the gelatin zymography, OPN ASO markedly decreased MMP-2 levels in HCCL6 cells. 
Table I. OPN ASO treatment reduces HCCLM6 cell lung metastasis in vivo.

\begin{tabular}{|c|c|c|c|c|}
\hline \multirow[b]{2}{*}{ Group } & \multirow[b]{2}{*}{ Tumor weight (g) } & \multirow{2}{*}{$\frac{\text { Intrahepatic metastasis }}{\text { Cases }(\%)}$} & \multicolumn{2}{|c|}{ Lung metastasis ${ }^{\mathrm{a}}$} \\
\hline & & & Cases $(\%)$ & No. of metastases \\
\hline OPN ASO & $1.5 \pm 0.5$ & $1 / 6(16.7)$ & $2 / 6(33.3)$ & $2.2 \pm 1.1$ \\
\hline Control ODN & $2.1 \pm 0.3$ & $3 / 6(50.0)$ & $6 / 6(100.0)^{\mathrm{b}}$ & $6.8 \pm 2.1^{\mathrm{b}}$ \\
\hline
\end{tabular}

${ }^{\text {aL }}$ Lung metastasis is denoted as both the cases of positive lung metastases and the average number of metastases in lungs per positive case $($ mean $\pm \mathrm{SD}) .{ }^{\mathrm{b}} \mathrm{P}<0.05$.
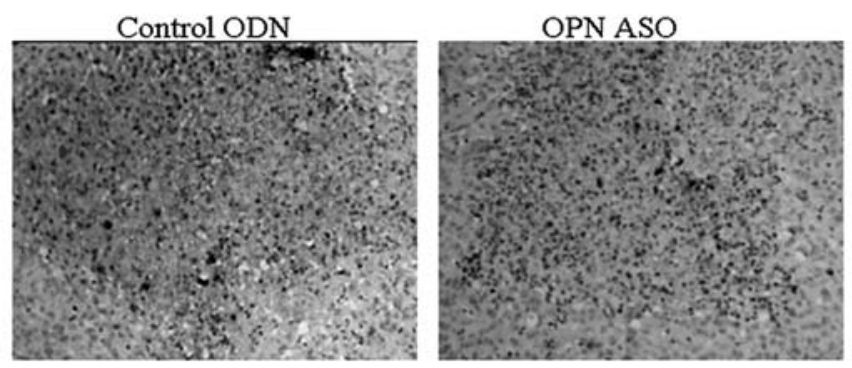

A

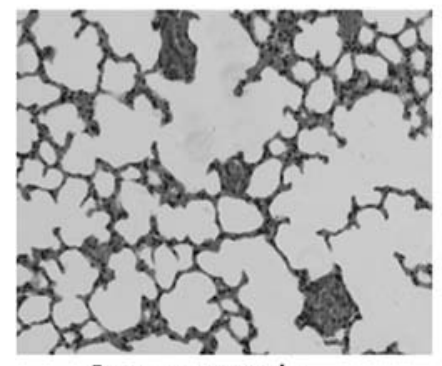

Lung metastasis

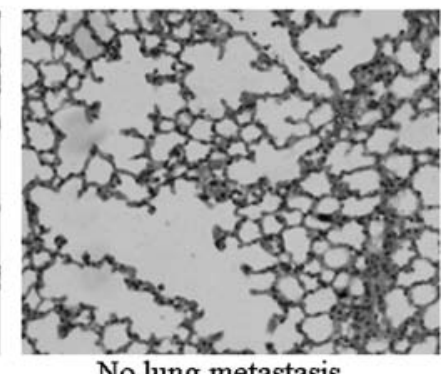

B

Figure 6. Inhibition of OPN expression in athymic mice bearing orthotopic HCCLM6 tumors following OPN ASO treatment. (A) OPN protein detected by immunohistochemistry was markedly reduced in intrahepatic tumor of OPN ASO-treated group (magnification, x50). (B) Representative lung tissue sections with metastatic lesions or no metastasis (hematoxylin and eosin; magnification, $\mathrm{x} 50$ ).

was due to reduced MMP-2, gelatin zymography was used to detect MMP-2 activity. The active form of MMP-2 was inhibited following OPN ASO treatment as indicated by the digestion of gelatin (Fig. 5B).

Therapeutic treatment with OPN ASO inhibits lung metastasis of orthotopically transplanted HCCLM6 tumors in nude mice. An advantage of the orthotopic HCCLM6 model with spontaneous lung metastasis mirrors clinical HCC progression and metastasis. We evaluated the therapeutic relevance of OPN ASO treatment in this model. Intraperitoneal injection of OPN ASO following orthotopic implantation inhibited OPN protein expression in HCC tumors (Fig. 6A). Tumor weight of OPN ASO-treated group was not significantly reduced compared with control oligonucleotide-treated group, but the incidence of lung metastasis in the animals treated with OPN ASO declined to $33.3 \%(2 / 6)$ from $100 \%(6 / 6)$ in control

oligonucleotide-treated animals $(\mathrm{P}=0.03$, examined by Fisher's exact test) (Table I) (Fig. 6B). In addition, the number of lung metastatic lesions in OPN ASO treatment group was also greatly decreased compared to control oligonucleotide-treated group (Table I).

\section{Discussion}

OPN expression has been linked to tumor progression and metastasis in a variety of human tumors including HCC (9). Many of the downstream signaling pathways and effectors including phosphotidylinositol 3-kinase (PI3K)/Akt, nuclear factor (NF)- $\mathrm{B}$, matrix metalloproteinases (MMPs) and uPA, are activated by OPN to facilitate tumor progression and targeting OPN signaling suppresses tumor progression in other types of cancers (10-13). As for HCC, OPN has been identified as the lead gene overexpressed in the metastatic HCC (3). Similar to other cancers, Sun et al (14) also found that OPN played an important role in HCC growth and metastasis through activating the mitogen-activated protein kinases (MAPK), NF-кB pathways and MMP-2. These findings indicate that OPN plays an important role in $\mathrm{HCC}$ progression and metastasis, and OPN might be a novel drug target for therapeutic intervention.

Human HCC cell line HCCLM6 features high lung metastatic potential and resembles typical cases of HCC in the clinic. OPN protein was overexpressed in HCCLM6 cells compared with non-metastatic HCC cell lines, suggesting OPN expression is associated with the highly metastatic potential. In the present study, suppression of OPN by 2'-MOE modified ASO inhibited malignant behaviors of HCCLM6 in vitro as well as in xenograft models. Cell migration and invasive capability of HCCLM6 cells following OPN knockdown by ASO were decreased and lung metastases were significantly reduced, supporting that the excessive expression of OPN is required for malignant phenotypes of HCC and OPN might be a new drug target for HCC progression and metastasis. Furthermore, the production of MMP-2 and uPA was reduced following the inhibition of OPN, suggesting MMP-2 and uPA might be the important mediators of OPN promoting $\mathrm{HCC}$ metastasis. This result is similar to that previously described (15). Mi et al (15) have demonstrated that OPN mediates metastatic behavior of murine mammary cancer through integrin-linked kinase-dependent activator protein-1 (AP-1) activation to up-regulate MMP-2 and uPA expression. 
RNA interference is an effective method for a specific gene knockdown (16), ASO has been used for decades for clinical translations from basic research (17). Vitravene, an ASO drug, is already marketed and approximately 20 antisense drugs are in clinical development, thus, it appears that antisense drugs may prove of value in the disease treatment (18). Since most of HCC are associated with chronic liver disease (19), it is preferable to use a highly target-specific drug approach to treat HCC. In this study, the ASO-mediated specific suppression of OPN expression in HCC cells was shown by oligonucleotide concentration- and treatment time-dependency, as well as sequence-specificity. It has been shown that 2'-MOE ASO can accumulate in the liver following systemic administration in mice (20). The results of in vivo orthotopic HCC models showed that ASO displayed significant suppression of OPN expression in tumor tissue.

In summary, our results demonstrate that the ASO targeting OPN significantly suppressed HCC cells invasion in vitro and effectively inhibited lung metastasis in orthotopic HCC model in vivo, suggesting targeting OPN might have a therapeutic benefit for patients to prevent HCC metastasis.

\section{Acknowledgements}

This study was supported by grant from the State Key Basic Research Program of China (No. 2004CB518708).

\section{References}

1. Parkin DM, Bray F, Ferlay $\mathrm{J}$ and Pisani P: Global cancer statistics, 2002. CA Cancer J Clin 55: 74-108, 2005.

2. Tang ZY, Ye SL, Liu YK, Qin LX, Sun HC, Ye QH, Wang L, Zhou J, Qiu SJ, Li Y, Ji XN, Liu H, Xia JL, Wu ZQ, Fan J, Ma ZC, Zhou XD, Lin ZY and Liu KD: A decade's studies on metastasis of hepatocellular carcinoma. J Cancer Res Clin Oncol 130: 187-196, 2004.

3. Ye QH, Qin LX, Forgues M, He P, Kim JW, Peng AC, Simon R, Li Y, Robles AI, Chen Y, Ma ZC, Wu ZQ, Ye SL, Liu YK, Tang ZY and Wang XW: Predicting hepatitis virus-positive metastatic hepatocellular carcinomas using gene expression profiling and supervised machine learning. Nat Med 9: 416-423, 2003.

4. Coppola D, Szabo M, Boulware D, Muraca P, Alsarraj M, Chambers AF and Yeatman TJ: Correlation of osteopontin protein expression and pathological stage across a wide variety of tumor histologies. Clin Cancer Res 10: 184-190, 2004.
5. Shevde LA, Das S, Clark DW and Samant RS: Osteopontin: an effector and an effect of tumor metastasis. Curr Mol Med 10: 71-81, 2010.

6. Wai PY and Kuo PC: Osteopontin: regulation in tumor metastasis. Cancer Metastasis Rev 27: 103-118, 2008.

7. Johnston NI, Gunasekharan VK, Ravindranath A, O'Connell C, Johnston PG and El-Tanani MK: Osteopontin as a target for cancer therapy. Front Biosci 13: 4361-4372, 2008.

8. Li Y, Tian B, Yang J, Zhao L, Wu X, Ye SL, Liu YK and Tang ZY: Stepwise metastatic human hepatocellular carcinoma cell model system with multiple metastatic potentials established through consecutive in vivo selection and studies on metastatic characteristics. J Cancer Res Clin Oncol 130: 460-468, 2004.

9. Rittling SR and Chambers AF: Role of osteopontin in tumour progression. Br J Cancer 90: 1877-1881, 2004.

10. Das R, Mahabeleshwar GH and Kundu GC: Osteopontin stimulates cell motility and nuclear factor kappaB-mediated secretion of urokinase type plasminogen activator through phosphatidylinositol 3-kinase/Akt signaling pathways in breast cancer cells. J Biol Chem 278: 28593-28606, 2003.

11. Das R, Philip S, Mahabeleshwar GH, Bulbule A and Kundu GC: Osteopontin: Its role in regulation of cell motility and nuclear factor kappa B-mediated urokinase type plasminogen activator expression. IUBMB Life 57: 441-447, 2005.

12. Robertson BW and Chellaiah MA: Osteopontin induces betacatenin signaling through activation of Akt in prostate cancer cells. Exp Cell Res 316: 1-11, 2010.

13. Castellano G, Malaponte G, Mazzarino MC, Figini M, Marchese F, Gangemi P, Travali S, Stivala F, Canevari S and Libra M: Activation of the osteopontin/matrix metalloproteinase-9 pathway correlates with prostate cancer progression. Clin Cancer Res 14: 7470-7480, 2008.

14. Sun BS, Dong QZ, Ye QH, Sun HJ, Jia HL, Zhu XQ, Liu DY, Chen J, Xue Q, Zhou HJ, Ren N and Qin LX: Lentiviralmediated miRNA against osteopontin suppresses tumor growth and metastasis of human hepatocellular carcinoma. Hepatology 48: 1834-1842, 2008.

15. Mi Z, Guo H, Wai PY, Gao C and Kuo PC: Integrin-linked kinase regulates osteopontin-dependent MMP-2 and uPA expression to convey metastatic function in murine mammary epithelial cancer cells. Carcinogenesis 27: 1134-1145, 2006.

16. Oh YK and Park TG: siRNA delivery systems for cancer treatment. Adv Drug Deliv Rev 61: 850-862, 2009.

17. Tamm I, Dorken B and Hartmann G: Antisense therapy in oncology: new hope for an old idea? Lancet 358: 489-497, 2001.

18. Wacheck V and Zangemeister-Wittke U: Antisense molecules for targeted cancer therapy. Crit Rev Oncol Hematol 59: 65-73, 2006.

19. But DY, Lai CL and Yuen MF: Natural history of hepatitisrelated hepatocellular carcinoma. World J Gastroenterol 14: 1652-1656, 2008.

20. Yu XX, Murray SF, Pandey SK, Booten SL, Bao D, Song XZ, Kelly S, Chen S, McKay R, Monia BP and Bhanot S: Antisense oligonucleotide reduction of DGAT2 expression improves hepatic steatosis and hyperlipidemia in obese mice. Hepatology 42: 362-371, 2005. 